\title{
Torri e piazzeforti nella Sardegna moderna. Fonti cartografiche e documentarie nella lettura delle trasformazioni territoriali del paesaggio costiero sardo tra XVI e XVIII secolo
}

\author{
Sebastiana Nocco \\ CNR-Istituto di Storia dell’Europa Mediterranea, Cagliari, Italia, nocco@isem.cnr.it
}

\begin{abstract}
In early modern, military engineers and architects of the Crown of Spain were engaged in the preparation of new fortifications systems to defend Mediterranean cities and coasts of raids from North. During the sixteenth century, the coastal landscape of the western Mediterranean underwent a profound change: major and minor cities were equipped with defensive walls and bastions; also around the coastal perimeter was built a system of circular watchtowers. The Kingdom of Sardinia, though with delay, was equipped with powerful defensive devices. Their phases of planning and realization can be partially reconstructed through working notes, reports, design drawings, maps and other important documents kept in the major European archives. The cartographic evidence on Sardinia - made from the second half of the sixteenth century to the beginning of the eighteenth century - record in some cases the presence of coastal towers existing and planned, offering interesting data.
\end{abstract}

Keywords: cartografía, Cerdeña, Edad Moderna / cartography, Sardinia, modern age.

\section{Introduzione}

Agli inizi dell'età moderna ingegneri e architetti militari al servizio della Corona di Spagna furono impegnati nella predisposizione di nuovi sistemi di fortificazioni idonei a difendere $\mathrm{i}$ centri litoranei e le coste mediterranee dalle incursioni barbaresche.

Nel corso del Cinquecento il paesaggio costiero delle due sponde del Mediterraneo subì così un profondo mutamento. I centri urbani furono fortificati con possenti mura e bastioni; lungo perimetro litoraneo si edificò, invece, un sistema di torri circolari di avvistamento, per lo più situate su alture o punte dalle quali era possibile godere di un'ampia visuale. Per affrontare al meglio i momenti di pericolo era fondamentale, infatti, che i segnali trasmessi da una torre fossero visibili dalle due più vicine su entrambi $i$ lati, in modo da attivare nel minor tempo possibile una catena di trasmissione ininterrotta dell'allarme.

La fortificazione delle frontiere della monarchia iberica avvenne in tempi diversi nei vari territori della Corona, dall'area iberica, ai possedimenti italiani peninsulari e insulari, ai Paesi Bassi, alle Indie.

Una delle prime esigenze che si avvertirono nello stato moderno fu, infatti, la rilevazione dei confini e il controllo delle frontiere, che imponeva ai sovrani una buona conoscenza dei propri territori, nonché l'elaborazione di progetti per la difesa militare che hanno lasciato ampie tracce nella produzione cartografica. La carta geografica è sempre stata, fin dall'antichità, uno strumento di potere indispensabile per il governo del territorio preziosa per la rilevazione e il controllo, ma anche per l'organizzazione e il 
dominio. Un legame che si fa ancora più evidente nella prima età moderna, allorché lo sviluppo della geografia è strettamente connesso con la formazione e il potenziamento dello stato moderno. Nel corso della sua storia la carta ha sempre svolto anche la funzione simbolica di "ritratto di un territorio" conquistato o da conquistare, ma soprattutto segno di un dominio.

\subsection{L'organizzazione della difesa nella Sardegna spagnola}

La Sardegna, per la sua posizione centrale, nei secoli basso-medievali era stata un importante crocevia e una tappa fondamentale nelle rotte mediterranee, ma oramai da qualche tempo le sue coste erano meta privilegiata delle scorrerie dei pirati barbareschi, divenendo terra di frontera in un Mediterraneo divenuto esso stesso teatro di guerra, "frontiera nel senso esatto del termine".

La cristianità, per far fronte ai nuovi pericoli, scruta le intenzioni del nemico utilizzando spie; invia in missione $\mathrm{i}$ propri ingegneri e architetti per predisporre la difesa delle aree strategiche, in particolare delle isole; munisce le coste di torri di guardia e segnali luminosi; erige muraglie e arsenali per respingere gli attacchi dell'artiglieria che hanno reso più vulnerabili le difese tradizionali.

L'avvento dell'artiglieria impose anche in Sardegna un adeguamento degli apparati difensivi esistenti. Fin dai primi del Cinquecento architetti e ingegneri militari della Corona furono impegnati della progettazione di cinte urbane bastionate e fortificate "alla moderna", nella convinzione che per mantenere il controllo di tutto il Regno fosse indispensabile conservare e potenziare Cagliari e Alghero, le città chiave della Sardegna spagnola.

L'inadeguatezza dei presidi difensivi del Regno sardo rispetto al potenziale militare dei turco-barbareschi era noto già da tempo alla Corona. Ancora nel tardo Quattrocento la difesa isolana continuava ad essere affidata a sistemi medioevali basati su cortine murarie e alti torrioni a protezione delle città più importanti.
Gli interventi di ammodernamento delle città più importanti, che furono assai intensi nei primi ottant'anni del Cinquecento, sono ben documentati nelle fasi iniziali, in cui operarono importanti personalità.

Tra essi grande importanza è stata finora attribuita all'attività ventennale del cremonese Rocco Capellino, inviato in Sardegna dall'imperatore Carlo V nel 1552 con l'ordine di procedere al riordino e al completamento delle fortificazioni del Regno, la cui professionalità è stata ridimensionata dagli studi più recenti e il cui ruolo attende di essere meglio chiarito. Varie circostanze inducono infatti a ritenerlo una figura minore, che diresse in loco i lavori progettati da un ingegnere di chiara fama rimasto anonimo. L'opera del Capellino è documentata da vari materiali topografici relativi ai piani delle fortificazioni da lui elaborati per alcune città isolane.

Nel 1563 Filippo II gli affiancò l'ingegnere Jacopo Palearo Fratino che predispose un nuovo progetto della cui realizzazione - sebbene con grosse modifiche - si occupò oltre un decennio più tardi il fratello Giorgio. Agli inizi del Seicento, dopo oltre vent'anni dalla partenza di quest'ultimo, gli interventi sulle piazzeforti sarde si ispiravano ancora ai progetti dei due fratelli.

Nel corso del XVII secolo diversi personaggi, ma per lo più figure minori, predisposero piani di intervento per fortificare le città isolane, spesso non recepiti dai governanti. La documentazione relativa a questo periodo, conservata nei diversi archivi italiani e iberici, è piuttosto frammentaria e necessita di ulteriori approfondimenti al fine di ricostruire un quadro più dettagliato, ma ci ha trasmesso i nomi di alcuni progettisti. Tra essi ricordiamo il viceré Juan Vivas (1622-1625) al quale sono attribuiti alcuni disegni relativi alle fortificazioni isolane.

Mentre sono in generale ben documentate le fasi relative agli interventi nelle piazzeforti, meno chiare sono le fasi iniziali della costruzione delle torri costiere, con lacune nella datazione dei manufatti più antichi, almeno fino all'istituzione per iniziativa di Filippo II della Reale Amministrazione delle Torri, con il compito di 
organizzare e gestire il sistema difensivo costiero.

Nella prima metà del Cinquecento le torri cittadine esistenti nelle principali piazzeforti sarde erano utilizzate con funzioni di vedetta: San Pancrazio di Cagliari, Castell'Aragonese, Longosardo, Terranova, Iglesias, Bosa e Porto Torres. Allo stesso tempo inizia l'edificazione delle prime torri costiere, avviata su iniziativa delle città regie, di imprenditori o signori feudali a tutela dei loro interessi. Nel 1518 la città di Sassari chiese di essere autorizzata a costruire una torre in Isla Llana; nel 1519 i corallari di Bosa costruirono una torre sulla foce del Temo e negli anni seguenti elevarono tre torri nella zona di Porto Ferro, a protezione del porto di imbarco. Sempre con tre torri venne fortificato anche il porto di Alghero; nel 1542 fu iniziata la torre "grande" di Oristano, la cui costruzione si protrasse diversi anni; mentre nel 1554 venne creato un fortilizio nel porto di Arbatax.

Nello stesso periodo furono inoltre attivati lungo le coste numerosi posti di guardia a carico delle città regie: Cagliari sorvegliava il tratto da Capo Carbonara a Sarroch; Iglesias da Capo Teulada a Capo Frasca; Oristano si occupava del Sinis; Bosa delle coste tra Santa Caterina di Pittinuri e Capo Marrargiu; da qui fino a Porto Ferro la difesa del territorio era di competenza di Alghero, mentre il controllo del litorale da Porto Ferro fino a Sorso (comprese le isole Piana e Asinara) ricadeva su Sassari; infine Castell'Aragonese si faceva carico del costo delle guardie in Anglona.

\section{Fonti documentarie e cartografiche}

Con l'avanzare della minaccia islamica l'interesse della monarchia ispanica si concentra maggiormente sul perimetro costiero, vero e proprio confine naturale, per la cui tutela era indispensabile provvedere con urgenza. La conoscenza del territorio e la sua rappresentazione grafica costituirono un momento importante in questa fase progettuale.

L'isola necessitava innanzi tutto di una nuova base cartografica, dato che le rappresentazioni all'epoca disponibili, ancora fortemente condizionate dalla geografia tolemaica, privilegiavano la piccola o la piccolissima scala, poco attenta al territorio e alle sue peculiarità geografiche.

Nella seconda metà del secolo XVI la cartografia della Sardegna si caratterizza per la quasi totale assenza di rappresentazioni sia letterarie, sia cartografiche riconducibili direttamente o indirettamente al governo spagnolo. Fatta eccezione per le topografie e piante delle città da fortificare, si contano pochi documenti cartografici.

$\mathrm{Su}$ iniziativa del viceré Juan Coloma, il pictor loci genovese Geronimo Ferra/Ferrua, realizzò tra il 1570 e il 1575 - dopo aver viaggiato a lungo in Sardegna insieme ad alcuni aiutanti una "pittura", accompagnata da una "descripcion y cosmographia" della Sardegna purtroppo perdute.

Nel 1572 il viceré Coloma aveva inoltre incaricato il capitano di Iglesias, Marco Antonio Camós, di compiere il periplo dell'isola per identificare i siti da fortificare. La relazione del Camós documenta l'esistenza di numerosi posti di guardia e di quindici torri già operative: Sant'Elia, Arbatax, Terranova, Porto Torres, Torre Antigua (presso Vignola), Monte Girat, Torre de Gainge, Portichol, Peña Maestra, Capo Giglio, Poglina, Bosa, Canjanas, Gran torre di Oristano e Cagliari. Risultavano invece in costruzione le torri di Columbargia, Capo Galera e delle saline della Nurra, mentre non è menzionata la torre dell'isola Piana, edificata mezzo secolo prima dai sassaresi e forse già abbandonata.

Negli anni più recenti, il ritrovamento presso l'Archivo General di Simancas di un disegno inedito della Sardegna, anonimo e non datato, ha consentito di gettare nuova luce sulle problematiche connesse alla predisposizione dei primi progetti relativi alla difesa costiera sarda. La carta, nella quale sono posizionate le torri costiere, sarebbe, infatti, anch'essa opera del Camós e conferma sostanzialmente i dati forniti dalla relazione.

Prima della pubblicazione di questo preziosissimo documento, la prima attestazione 
della presenza delle torri costiere sarde nella cartografia era comunemente considerata la carta corografica realizzata da Rocco Capellino dopo vent'anni di rilevamenti in loco. In tale circostanza egli si era reso conto che «... el disegno de dita isola non è mai stato tirato ala sua bona forma et siando io stato et praticato da venti hani in far fortificar quei lochi per ordine di sua mag. ${ }^{\text {tà }}$, non ho volsuto manchar de retrarla in la melio forma che si è potuto...». Malgrado $\mathrm{i}$ buoni propositi, la carta mostra una grande imprecisione, che non le impedì però di fungere da "modello forte" e - grazie all'avvallo datole dal cartografo padovano Giovanni Antonio Magini - esercitò la sua influenza per oltre un secolo e mezzo.

Tuttavia essa non contribuí a una migliore conoscenza del territorio isolano, né tantomeno delle sue coste, nonostante il contemporaneo impegno del Capellino nella rilevazione e progettazione delle fortificazioni "alla moderna" delle più importante piazzeforti isolane.

La carta dell'ingegnere cremonese, nelle sue tre redazioni disegnate a Roma nel maggio 1577, è costellata lungo il profilo costiero di tante piccole torrette cilindriche disegnate in prospettiva, la cui sequenza, ubicazione e quantità differisce nelle tre stesure.

La schedatura dei toponimi citati in relazione alle torri costiere offre dati molto differenti nelle tre redazioni della carta, passando da una quindicina nella redazione $\mathrm{A}$, a oltre cinquanta nella redazione C. Il gran numero di torri segnalate impone un'immediata riflessione, dato che la documentazione non conferma l'esistenza di molti di questi manufatti già nel 1577. Ricordiamo inoltre che, sebbene sia possibile accettare tale anno per la realizzazione della carta, ritenendola coeva alla Relazione, assai ampio rimane l'arco cronologico nel quale il Capellino ha effettuato la raccolta dei dati, che si colloca nel ventennio 1552-1572. A ciò si aggiunga il fatto che finora non è stato possibile ricostruire nel dettaglio le date e gli itinerari dei suoi spostamenti nell'isola, al fine di individuare con maggiore precisione il periodo cui si riferisce la situazione rappresentata in ciascuna delle tre carte.

La realizzazione della carta del Capellino si colloca in un momento molto delicato della discussione in atto nell'isola sull'opportunità e le modalità in cui edificare le torri costiere. Non a caso proprio in quegli anni Camós elaborava dettagliate relazioni sullo stato dei luoghi e sugli interventi necessari e una carta da inviare a Filippo II.

Una corretta interpretazione della carta del Capellino non può prescindere dunque dal confronto con la relazione già citata del Camós, e con la carta custodita nell'Archivio di Simancas, dalle quali emerge un quadro preciso e dettagliato dello stato delle fortificazioni isolane nel 1572.

Questi pochi elementi ci consentono già di avanzare ragionevoli dubbi sulle torri ubicate dal Capellino, il quale, a nostro avviso, ha voluto segnalare con il disegno della torretta sia i posti di guardia, sia le torri realmente esistenti, adottando un unico simbolo per indicare un apprestamento difensivo in genere. Ma anche ammettendo questa ipotesi, non mancano le imprecisioni. Prova ne sarebbe anche il fatto che nella redazione $\mathrm{C}$ figura una torre nell'estremità di ciascun promontorio della costa orientale, da «congiano» (antico porto fortificato dove non è mai esistita una torre) fino a «capo carbonara» (dove la città regia di Cagliari pagava nel 1572 guardie a piedi, poiché la torre è più tarda). In effetti l'interesse principale del Capellino cartografo è lo stesso che aveva guidato l'azione del Capellino ingegnere, ossia la fortificazione delle piazzeforti e non dei litorali. Nel delineare la carta corografica, invece, il Capellino sembra abbandonare la veste ufficiale e dedicarsi ad una impresa che, lo ricordiamo, era frutto dell'iniziativa personale e non era destinata a rimanere segreta, come avrebbe imposto il suo ruolo di ingegnere al servizio del sovrano, ma pensata per la stampa.

Questo non avvenne, ma la redazione B della carta, non orientata, dovette trovare comunque immediata diffusione a Roma e secondo alcuni studiosi costituirebbe l'archetipo dell'immagine della Sardegna dipinta nella Galleria del 
Belvedere in Vaticano dal frate domenicano Egnatio Danti nel 1581. Diversi elementi accomunano, infatti, le due carte: la forma dell'isola fortemente allungata in direzione Nord/Sud, frastagliata da promontori e golfi con profonde insenature a rias che ne deformano il profilo costiero; il golfo e la città di Cagliari orientati a Sud-Est; la presenza delle torri costiere.

In relazione a queste ultime, però, il Danti ha seguito in gran parte la redazione B di Capellino (pur recependo un numero inferiore di torri), ma ne ha anche aggiunto tre presenti, invece, solo nella cosiddetta redazione $C$ : due senza nome nella costa orientale e una ubicata a «Maifera». Le informazioni sulle torri non sono state accolte invece nella rielaborazione che delle carte del Capellino fece Giovanni Antonio Magini.

Il dipinto di Egnatio Danti ripropone dunque la sequenza di torri proposta dal Capellino, ma ne cura maggiormente la rappresentazione grafica, operando una scelta dettata da una ricerca di gradevolezza dell'immagine. Per questo alterna piccoli prospetti di torri circolari e quadrate, alcune anche fortificate da una cinta muraria, senza che ci sia però una distinzione effettiva, né una conoscenza diretta dei luoghi. Superfluo dire che, rispetto al Capellino, nessun aggiornamento compare nella carta del Danti relativamente alle torri che nel frattempo erano state già costruite $\mathrm{o}$ almeno iniziate. Negli anni che intercorrono tra la stesura delle carte dell'ingegnere cremonese e le pitture della Galleria del Belvedere, infatti, si erano verificati alcuni avvenimenti che ebbero importanti ripercussioni sugli equilibri europei.

In seguito alla sconfitta della Goletta, infatti, avvenuta come noto nell'estate del 1574, la Spagna era stata costretta a ripensare la propria strategia difensiva partendo da una revisione generale delle fortezze. Così, mentre nel gennaio 1575 Juan Çanoguera effettuava una indagine sullo stato delle difese isolane e in particolare delle piazzeforti di Cagliari e Alghero, tre anni dopo il viceré de Moncada imprimeva una svolta con la propria azione di governo, predisponendo un piano di difesa globale, studiato per garantire a lungo termine la sicurezza dei litorali.
In accordo con Filippo II decise di approntare una milizia che intervenisse su segnalazione di un circuito di torri d'avvistamento simile a quello costruito nei regni di Valenza, Napoli e Sicilia, affidando a città e baroni la fortificazione delle località che erano soliti far presidiare da gruppi di sentinelle armate, mentre i rimanenti tratti di litorale sarebbero stati di competenza dell'Amministrazione delle torri, sotto la cui egida si completò il sistema.

Assai utile per comprendere l'assetto difensivo isolano nell'ultimo ventennio del Cinquecento è un documento datato 26 giugno 1580, nel quale il de Moncada scriveva al sovrano per informarlo che nelle marine di Ogliastra, Posada e Gallura erano sempre attivi i posti di guardia, mentre sarebbe occorso costruire un paio di torri nelle Bocche di Bonifacio (comunque insufficienti per un territorio che si estendeva fino a Castell'Aragonese). Alla città di Sassari era stato imposto un pagamento per la riparazione delle mura, mentre il suo territorio e la marina erano protetti da due torri molto importanti, una delle quali era già terminata $\mathrm{e}$ l'altra lo sarebbe stata entro un mese. Nella marina di Alghero risultava invece finita la torre del Capo della Galera e se ne stava costruendo una nuova nei territori posseduti nel litorale dai vassalli della baronia di Monteleone, i quali pagavano anche la guardia della costa.

Il viceré sottolineava inoltre che a Bosa sarebbe stato necessario intervenire sulle muraglie, costruire una fortezza sopra il Monte de Austria e riparare la torre che stava nell'isoletta a difesa del porto (ci sarebbe voluto un anno per rimetterla a posto). Si stavano ultimando invece le due torri che insistevano su questo tratto costiero e le altre due ubicate nei territori di don Angelo Setrillas, con la speranza di terminarle presto e dotarle di guardie.

Ad Oristano, nel cui porto esisteva già una torre, si procedeva alla riparazione delle mura e alla conclusione delle due torri iniziate, mentre si era ordinato di edificarne un'altra. A Iglesias risultava terminata la torre iniziata l'anno precedente e si stavano completando le dieci torri che erano state proposte nel golfo di Cagliari. I baroni con i loro cavalli, inoltre, 
avrebbero dovuto prestare servizio di guardia come aveva ordinato sua Maestà e secondo il parere del Fratino (Jacopo).

Nell'ultimo quindicennio del XVI secolo la Chorographia Sardiniae del sassarese Giovanni Francesco Fara contribuisce ad un quadro fedele della situazione in quegli anni: infatti, nel descrivere il periplo dell'isola, segnala le torri esistenti e suggerisce l'edificazione di nuove, forse sulla base delle indicazioni desunte dalle numerose relazioni stilate in quegli anni.

Egli inizia la propria descrizione partendo dalla costa settentrionale, e precisamente dal promontorio noto a Tolomeo, Plinio e Marziano Capella come Gorditano, nel quale si trovava anche l'osservatorio di Monte Falcone, che meriterebbe, a detta dell'autore, di essere dotato di una solida torre, peraltro già segnalata nelle carte del Capellino come esistente.

La prima torre nel litorale settentrionale è quella delle Saline di Sassari, seguita da quella antica nel promontorio Russu, mentre tra le due situa il porto fortificato di Porto Torres. Nella costa orientale lo storico sassarese ricorda il «portum congianum tutissimum», ben riparato e in grado di accogliere numerose imbarcazioni, e due torri, una nei pressi del porto di Terranova, l'altra nel promontorio di Arbatax.

La costa meridionale si presentava all'epoca già ben torreggiata: si menzionano quella di Porto Giunco nel Capo Carbonara, Cala Caterina, la Rocca Vecchia (ubicata subito dopo la foce del fiume Carbonara), seguite dalle torri di Capo Boi, Capo Vacca (o Monte Fenugu), Guardiana (detta anche di Cala Serraina) e Noraxia. Sempre sul versante meridionale si segnalano quelle di Fogue de Olla del fiume di Sant'Andrea (detta anche Pie' d'Altare), Carcagnolu e, nel paese di Quartu, quella detta "della foce del fiume".

A Capo Sant'Elia si trovavano, oltre alla torre omonima, quelle di Cala Mosca e di Perda Liana. Tra il Capo Sant'Elia e il Capo di Pula è il promontorio cagliaritano, ove sono Cagliari e il suo vastissimo porto in grado di dare rifugio a flotte di grande entità. Sul lato opposto era presente la torre di Solvi (detta di Santa Maddalena), quella di Antigori ubicata nella località "Fiume del vino", mentre in una grande altura era quella di Sorba. Nel promontorio detto Cunicularium da Tolomeo, ora Capo di Pula, nel quale giace anche la città distrutta di Nora, il Fara ubica infine la torre chiamata "osservatorio di Bruto". Passando alla costa occidentale, l'elenco prosegue con la torre di Arenella, edificata a protezione di un vastissimo porto nella foce del Flumentorgiu; la ben fortificata Torre Grande di Oristano, a difesa del golfo; quella sul promontorio di San Marco, recentemente eretta e, a 6 miglia da essa, l'antica torre di Corriga, alquanto in rovina.

Seguivano quella di Capo Mannu o delle Saline e di Cala di Capo Bianco, presso le saline di Oristano. Una torre di avvistamento era a Cala Puttu, una a Santa Caterina posta a tutela di un ottimo punto di approdo per piccole imbarcazioni e ancora le torri di Punta Negra, della foce del fiume Fogue de Olla, di Liscia Rossa e di Columbargia o Fiscella, ove sono due piccoli scali. La foce del fiume Temo di Bosa era protetta da una torre situata su un'isola, mentre le cale vicine erano sorvegliate dalle torri di Punta Argentina e del promontorio Marrargiu, cui facevano seguito quella della Cala e porto di Poglina e dell'Uomo Morto. Il golfo e il porto di Alghero erano presidiati dalla torre di Capo Galera, mentre l'accesso a Porto Conte era protetto da quella di Capo Giglio, non lontano dal quale sorgeva una roccaforte da poco eretta. Chiudono, infine, il periplo dell'isola la torre Primaria o Tor Maestra, quella del porto di Porticciolo, di Cala Viola e quella nei pressi della cala Mont Girat, nel cui mare si pesca il corallo.

Tra il 1584 e il 1621 furono costruite altre quarantasei torri, ma non tutte erano in servizio contemporaneamente. Esse avevano raggiunto il numero di un'ottantina nel primo trentennio del secolo, quando risultano inserite nella cosiddetta carta "sardo-spagnola" che si conserva nella Biblioteca Nazionale di Parigi. La sua realizzazione sarebbe stata promossa dal magistrato sassarese Francisco Vico e stampata in un'unica copia, forse in Spagna, intorno al 1639 , anno in cui venne pubblicata a Barcellona la Historia general de la Isla y Reyno de 
Sardeña dello stesso Vico. Infatti, quest'ultimo potrebbe avere realizzato in prima persona la carta, servendosi dei materiali cartografici esistenti nel Consiglio d'Aragona presso la Corte di Madrid, istituzione in cui egli operò, e in particolare potrebbe essersi avvalso proprio del disegno del pittore genovese Girolamo Ferrua, cui abbiamo fatto cenno in precedenza.

Più agevole ricostruire in questo documento le torri costiere, dato che a quest'epoca il sistema era quasi completato e la carta si presenta generalmente precisa nel riportare le torri esistenti (rappresentate con un piccolo disegno prospettico e tutte con pianta circolare), accompagnate nella gran parte dei casi dal nome, con poche eccezioni.

Qualche incertezza mostra tuttavia l'ubicazione di alcune torri: nella parte meridionale dell'isola è segnalata la torre di San Miguel e quella «de La isla de los coles», ma i due toponimi si riferiscono alla stessa torre, di San Michele nell'isola dei Cavoli. Allo stesso modo la torre «de Sarpentara» e quella di San Luis corrispondono allo stesso manufatto, poi dedicato al santo. La torre «de iana Safceme», invece, il cui toponimo corrisponde all'odierno Cala dei Corsari, propone il caso di una torre mai esistita, visto che il sito ospitava un posto di guardia, ma il toponimo è presente sempre nell'accezione di torre anche in Rocco Capellino.

Un altro dubbio mostra l'identificazione di due torri nel Sarrabus: la torre «de Orri», che potrebbe corrispondere a Zacurro, variante toponomastica con cui era nota la torre di S. Gemiliano di Tortolì, in costruzione nel primo quarto del Seicento; la seconda, «T. de P..nte Corala», che verrebbe spontaneo identificare con la torre Sarralà di Tertenia, la cui costruzione risulta però più tarda. Di origine incerta è anche la torre «de Cabo de Mosco», oggi del Sevo di Cabras, ma un tempo detta di Cala Moscas, che compare qui per la prima volta. Si sottolineano, infine, le assenze di alcune importanti torri del sistema cagliaritano, certamente già attive nel periodo, come testimoniano varie fonti.

\section{Conclusioni}

Con l'arrivo dei piemontesi, nel 1720, le difese isolane appaiono in condizioni precarie: ben presto gli ingegneri militari si occuperanno della riparazione e dell'ammodernamento delle piazzeforti di Cagliari e Alghero, nonché del ripristino delle torri costiere che versavano in stato di abbandono. Intorno al 1720 venne infatti realizzata la Carta del Litorale del Regno di Sardegna in cui si vedono le Torri esistenti e quelle di progetto, la cui attribuzione all'ingegner Felice de Vincenti non è ancora accertata. In epoca sabauda il sistema delle fortificazioni isolane veniva ancora potenziato con la realizzazione di una trentina di forti in località strategiche.

Le torri costiere della Sardegna persero definitivamente la loro funzione difensiva con il Regio Decreto del 25 aprile 1867, il quale elencò i castelli, le torri e le fortezze che cessavano di essere considerati come posti fortificati del nuovo Regno d'Italia.

Da allora inizia per le torri sarde un lento declino che giunge purtroppo fino ai nostri giorni. Solo la sensibilità di alcune amministrazioni "illuminate" che hanno realizzato progetti di recupero e, nei casi più felici, interventi di riuso sostenibile di questi edifici ne hanno consentito una fruizione ulteriore turistica, ma soprattutto culturale. Essenziale resta, in tutti i casi, per un recupero ottimale di questi manufatti, la loro conoscenza storica e geostorica, supportata dai più moderni metodi di indagine oggi offerti anche dall'archeologia post-medievale, affinché tutte le torri costiere sarde possano godere di una idonea e completa valorizzazione.

\section{References}

AA.VV. (1999). Imago Sardiniae. Cartografia storica di un'isola mediterranea. Consiglio Regionale della Sardegna. Cagliari, 1999. 
Alberti O. (1970). Le carte della Sardegna di Rocco Capellino, in «Nuovo Bollettino Bibliografico Sardo», XII; n. 70, pp. 3-9; n. 71, pp. 3-10; n. 72, pp. 3-7 e tavv. f. t.

Anatra B., Mele M. G., Murgia G., Serreli G. coord. (2008). "Contra Moros y Turcos". Politiche e sistemi di difesa degli Stati mediterranei della Corona di Spagna in Età Moderna. ISEM. Cagliari.

Baldacci O. (1973). Una carta geografica seicentesca della Sardegna in redazione spagnola, in «Rivista Geografica Italiana». LXXX, fasc. IV. pp. 369-388.

Cámara A. (1998). Fortificación y ciudad en los reinos de Felipe II. Nerea. Madrid.

Deledda S. (1936-40). La carta della Sardegna di Rocco Cappellino (1577), in "Archivio Storico Sardo», XX (1936), fascc. III-IV, pp. 84-121 e XXII (1939-40), fasc. I, pp. 27-48.

Hernando Sánchez C. J. coord. (2000). Las fortificaciones de Carlos V. Ediciones del Umbral. Madrid.

Kagan R.L., Schmidt B. (2007). "Maps and the Early Modern State. Official Cartography" in Woodward D. coord. The History of Cartography, vol. 3 Cartography in the European Renaissance. part 1. University of Chicago Press. Chicago \& London. pp. 661-679.

Mele G. (2000). Torri e cannoni. La difesa costiera in Sardegna nell'età moderna. EDES. Sassari.

Mele G. (2006). Documenti sulla difesa militare della Sardegna in età spagnola. Fondazione Banco di Sardegna. Sassari.

Montaldo G. (1992). Le torri costiere della Sardegna. Carlo Delfino. Sassari.

Nocco S. (2003). "Forma e rappresentazione delle città sarde in Età Moderna" in El món urbà a la Corona d'Aragó del 1137 als decrets de nova planta. Atti del XVII Congresso di Storia della Corona d'Aragona. Universitat de Barcelona. Barcellona. vol. II, pp. 817-830.

Nocco S. (2009). I progetti per le fortificazioni nella Sardegna moderna. in «RiMe. Rivista dell'Istituto di Storia dell'Europa Mediterranea», 2. pp. 131-141.

Nocco S. (2014). "La cartografia per la difesa del territorio" in Guia Marin L., Mele M.G., Tore G.F. coord. Identità e frontiere: politica, economia e società nel Mediterraneo (secoli XIV-XVIII). Franco Angeli. Milano. 2014. pp. 218-225.

Nordman D. (2008). "Frontières et limites maritimes: la Méditerranée à l'époque moderne (XVI ${ }^{\mathrm{e}}-\mathrm{XVIII}{ }^{\mathrm{e}}$ siècle)" in Fasano Guarini E., Volpini P. coord. Frontiere di terra, frontiere di mare. La Toscana moderna nello spazio mediterraneo. F. Angeli. Milano. pp. 19-34.

Pillosu E. (1959-1960). Un inedito rapporto cinquecentesco sulla difesa costiera della Sardegna di Marco Antonio Camos, in «Nuovo Bollettino Bibliografico Sardo», IV (1959), n. 21, pp. 3-10; n. 22 , pp. 7-12; n. 23, pp. 3-8; n. 24, pp. 3-7; V (1960), n. 25, pp. 5-9.

Principe I. (1982). "La Sardegna spagnola: cristallizzazione di una società periferica" in Il progetto del disegno. Città e territori italiani nell'Archivo General di Simancas. Casa del Libro. Reggio Calabria/Roma. pp. 189-199.

Putzulu E. (1955). Di una sconosciuta Cosmografia cinquecentesca della Sardegna in «Nuovo Bollettino Bibliografico Sardo». I, 1. pp. 8-9.

Rassu M. (2005). Sentinelle del mare. Le torri della difesa costiera della Sardegna. Grafiche del Parteolla. Dolianova.

Tenenti A. (1994). "Problemi difensivi del Mediterraneo nell'età moderna" in Mattone A., Sanna P. coord. Alghero, la Catalogna, il Mediterraneo. Storia di una città e di una minoranza catalana in Italia (XIV-XX secolo). Gallizzi. Sassari. pp. 311-318

Viganò M. (2004). «El fratin mi ynginiero». I Paleari Fratino da Morcote ingegneri militari ticinesi in Spagna (XVI-XVII secolo). Casagrande. Bellinzona.

Zedda Macciò I. (2007). "Insularità e rappresentazione dello spazio costiero. Frontiere del Regnum Sardiniae nell'Età Moderna” in Conti S. coord. Amate sponde. Le rappresentazioni dei paesaggi costieri mediterranei. Grafica Art. Formia. pp. 313-355.

Zedda Macciò I. (2008). "Costruire la carta negli Stati della Corona di Spagna. Istruzioni centrali e applicazioni periferiche" in Masetti C. coord. Atti del Primo Seminario di Studi Dalla mappa al GIS (Roma, 5-6 marzo 2007). Brigati. Genova. pp. 63-114. 